\title{
Otimização multiobjetivo utilizando o NSGA-II adotando uma nova abordagem para reconfiguração na distribuição de sistemas de potência
}

\author{
Ênio R. Viana* Aldir Silva Sousa ${ }^{* *}$ \\ * Universidade Federal do Piauí \\ Departamento de Engenharia Elétrica - Centro de Tecnologia \\ Teresina, Piauí,Brasil, (e-mail: eniocc@gmail.com). \\ ** Universidade Estadual do Piauí \\ Centro de Tecnologia e Urbanismo \\ Teresina, Piauí, Brasil (e-mail: aldirsousa@gmail.com)
}

\begin{abstract}
The reconfiguration problem has the objective of finding the best radial topology in a Radial Distribution System (RDS) in order to, among other possible objectives, minimize the active power losses. This work proposes a new approach for the multiobjective reconfiguration of this type of network. The multiobjective reconfiguration in RDS's presents itself as a problem of combinatorial complexity, with numerous restrictions and diverse solutions. The reconfiguration technique is one of the most used because it is low cost, compared to other approaches to reduce technical losses. The proposed approach was tested using the NSGA-II multi objective Evolutionary Algorithm (AE) (Elitist Non-Dominated Sorting Genetic Algorithm). The approach proposed here was able to treat the infeasible solutions, specific to AE's, in the systems tested (33, 84 and 136 bars). It can be verified the efficiency and robustness of the methodology even for medium systems

Resumo: O problema de reconfiguração tem por objetivo encontrar a melhor topologia radial em um Sistema de Distribuição Radial (SDR), de modo a, dentre outros possíveis objetivos, minimizar as perdas de potência ativas. Este trabalho propõe uma nova abordagem para a reconfiguração multiobjetivo deste tipo de rede. A reconfiguração multiobjetivo em SDR's apresenta-se como um problema de complexidade combinatória, com inúmeras restrições e soluções diversas. A técnica de reconfiguração é uma das mais utilizadas por ser de baixo custo, frente a outras abordagens de redução de perdas técnicas. A abordagem proposta foi testada utilizando o Algoritmo Evolutivo (AE) multiobjetivo NSGA-II (Elitist Non-Dominated Sorting Genetic Algorithm). A abordagem aqui proposta foi capaz de tratar as soluções infactíveis, próprias de AE's, nos sistemas testados (33, 84 e 136 barras). Pode-se constatar a eficiência e robustez da metodologia mesmo para sistemas de médio porte.
\end{abstract}

Keywords: Power Distribution System, genetic algorithms, Non-dominated Sorting Genetic Algorithm, multi-objective optimization, Reconfiguration.

Palavras-chaves: Sistemas de Distribuição de Energia, Algoritmos Genéticos, Algoritmo Genético Ordenado por Não Dominância, Otimização Multiobjetivo, Reconfiguração.

\section{INTRODUÇÃO}

O problema de Reconfiguração de Sistemas de Distribuição Radial (RSDR) de energia elétrica tem por objetivo alterar a topologia da rede com o intuito de se alcançar diversos objetivos, entre eles: redução das perdas técnicas, priorização de cargas, balanceamento de alimentadores, isolamento de trechos durante falhas, etc.

Encontrar a melhor topologia para determinado objetivo é, muitas vezes, algo que demanda um grande esforço computacional, devido à natureza combinatória do problema de RSDR.
Com o crescimento das áreas urbanas e consequentemente, com o aumento das redes de distribuição de energia elétrica, a reconfiguração de redes, até então de porte pequeno, passou a necessitar de técnicas computacionais para tanto, deixando de lado todo o tratamento subjetivo e humano inerente a este problema.

Paralelamente ao crescimento dos sistemas de distribuição, cresceu a preocupação quanto à qualidade da energia fornecida aos consumidores. Com isto, criaram-se normas reguladoras para a distribuição de energia. Neste cenário, surgiu o PRODIST (Procedimentos de Distribuição de Energia Elétrica no Sistema Elétrico Nacional), que lista, em seu modulo I item 2, como um de seus objetivos a 
garantia de que os sistemas de distribuição devam operar em segurança, eficiência, qualidade e confiabilidade.

Por se tratar de um problema de caráter combinatório, as soluções clássicas de otimização não se apresentam como primeira opção na resolução do problema de reconfiguração. Heurísticas e meta-heurísticas são as abordagens mais utilizadas na literatura para a resolução do problema de RSDR, Su et al. (2005), Shojaeian and Ghandehari (2013) e Oliveira (2011).

Heurísticas de caráter evolutivo tem em sua natureza a geração e avaliação de indivíduos (soluções) de uma determinada população (espaço de busca) ao longo de épocas (iterações). Assim como na teoria na qual foi inspirada (Teoria Evolucionista de Charles Darwin), aqui os indivíduos também sofrem crossover e mutação (operadores genéticos).

A ação dos operadores genéticos sobre as soluções durante a evolução do algoritmo pode gerar soluções inviáveis na prática. Constantes avaliações destas soluções podem gerar atrasos na evolução do algoritmo e um maior esforço computacional na tentativa de fugir de pontos de ótimos locais.

Neste artigo, propõe-se uma heurística de factibilização dessas soluções ditas infactíveis, com o intuito de fornecer um menor esforço computacional na otimização de dois objetivos (redução da perda de potência ativa e redução do número de manobras necessárias para atingir uma configuração que atinja a perda de potência citada), além de atingir boas ou ótimas soluções para o Algoritmo Evolutivo (AE) NSGA-II (Elitist Non-Dominated Sorting Genetic Algorithm).

\section{FLUXO DE CARGA EM SISTEMAS DE DISTRIBUIÇÃO RADIAL}

No fluxo de carga de uma rede de energia elétrica determinam-se os estados de operação de uma rede, ou seja, calculam-se os valores de magnitude de tensão e ângulos de fase para uma determinada condição de geração e carga daquela rede.

Apesar de se apresentar como o método de fluxo de carga mais utilizado nas concessionárias de energia segundo Carvalho (2006), o método de Newton Raphson é adequado para sistemas malhados, característica não encontrada em SDR's, além do fato deste método utilizar-se do uso de uma matriz Jacobiana, o que foi decisivo para o não uso do mesmo devido ao alto custo computacional inerente aos cálculos de matriz inversa.

Neste estudo, foram utilizados SDR cuja característica malhada não existe em sua operação. Outra característica importante encontrada nos sistemas trabalhos é a relação resistência/reatância $(\mathrm{R} / \mathrm{X})$ alta. Estas duas características somadas ao alto custo computacional descartaram o uso do método de Newton Raphson.

O método de fluxo de carga utilizado foi o Backward Forward Sweep, Shirmohammadi et al. (1988). Neste método, inicialmente defini-se uma magnitude de tensão fixa para todas as barras do sistema, é comum o uso de um valor igual ou muito próximo ao da barra slack (barra de referência).
Fixadas as tensões nas barras, são calculadas todas as correntes dos ramos da rede, conforme Equação 1a e 1b.

$$
\begin{gathered}
{\left[\begin{array}{c}
I_{i a} \\
I_{i b} \\
I_{i c}
\end{array}\right]^{(k)}=\left[\begin{array}{c}
\left(S_{i a} / V_{i a}^{k-1}\right)^{*} \\
\left(S_{i b} / V_{i b}^{k-1}\right)^{*} \\
\left(S_{i c} / V_{i c}^{k-1}\right)^{*}
\end{array}\right]-Y V} \\
Y V=\left[\begin{array}{ccc}
Y_{i a}^{*} & 0 & 0 \\
0 & Y_{i b}^{*} & 0 \\
0 & 0 & Y_{i c}^{*}
\end{array}\right]\left[\begin{array}{l}
V_{i a} \\
V_{i b} \\
V_{i c}
\end{array}\right]^{(k-1)}
\end{gathered}
$$

Com $k$ representando a iteração atual; $I_{i a}, I_{i b}, I_{i c}$ são as injeções de corrente na barra $i ; S_{i a}, S_{i b}, S_{i c}$ são as injeções de potência na barra $i ; V_{i a}, V_{i b}, V_{i c}$ são as tensões na barra $i$; e $Y_{i a}, Y_{i b}, Y_{i c}$ são as admitâncias no nó $i$.

O processo de backward é iniciado das barras terminais indo em direção à subestação. A corrente na linha $i$ é representada por:

$$
\begin{gathered}
{\left[\begin{array}{c}
J_{l a} \\
J_{l b} \\
J_{l c}
\end{array}\right]^{k}=\left[\begin{array}{c}
V_{i a} \\
V_{i b} \\
V_{i c}
\end{array}\right]^{(k-1)}+I J} \\
I J=-\left[\begin{array}{ccc}
I_{j a} & 0 & 0 \\
0 & I_{j b} & 0 \\
0 & 0 & I_{j c}
\end{array}\right]+\sum_{m \in M}\left[\begin{array}{c}
J_{m a} \\
J_{m b} \\
J_{m c}
\end{array}\right]^{k}
\end{gathered}
$$

Com $J_{l a}, J_{l b}, J_{l c}$, as correntes na linha $l ; M$ o número de linhas conectadas na saída da barra $j$; e $J_{m a}, J_{m b}, J_{m c}$, as correntes na linha $m$.

O processo forward inicia-se da subestação em direção às barras terminais, atualizando as tensões nas barras conforme as equações $3 \mathrm{a}$ e $3 \mathrm{~b}$.

$$
\begin{gathered}
{\left[\begin{array}{c}
V_{j a} \\
V_{j b} \\
V_{j c}
\end{array}\right]^{k}=\left[\begin{array}{c}
V_{i a} \\
V_{i b} \\
V_{i c}
\end{array}\right]^{k}-Z J} \\
Z J=-\left[\begin{array}{lll}
Z_{a a, l} & Z_{a b, l} & Z_{a c, l} \\
Z_{b a, l} & Z_{b b, l} & Z_{b c, l} \\
Z_{c a, l} & Z_{c b, l} & Z_{c c, l}
\end{array}\right]\left[\begin{array}{c}
J_{l a} \\
J_{l b} \\
J_{l c}
\end{array}\right]^{k}
\end{gathered}
$$

Os passos até aqui representam apenas uma única iteração $k$. Finalizada uma iteração, calculam-se os mismatches (diferenças) de potência, conforme Equação 4.

$$
\begin{aligned}
\Delta S_{i a}^{(k)} & =V_{i a}^{(k)}\left(I_{i a}{ }^{(k)}\right)^{*}-Y_{i a}{ }^{*}\left|V_{i a}\right|^{2}-S_{i a} \\
\Delta S_{i b}^{(k)} & =V_{i b}^{(k)}\left(I_{i b}{ }^{(k)}\right)^{*}-Y_{i b}{ }^{*}\left|V_{i b}\right|^{2}-S_{i b} \\
\Delta S_{i c}^{(k)} & =V_{i c}^{(k)}\left(I_{i c}{ }^{(k)}\right)^{*}-Y_{i c}{ }^{*}\left|V_{i c}\right|^{2}-S_{i c}
\end{aligned}
$$

Se a parte real ou imaginária(potência ativa ou reativa) de qualquer um dos mismatches calculados for maior que o critério de parada $\epsilon$, uma nova iteração deve ser realizada até que a convergência seja alcançada, Shirmohammadi et al. (1988).

\section{ALGORITMO NSGA-II}

O problema de RSDR tem caráter multimodal e com uma possibilidade de soluções de natureza combinatória, 
tornando as técnicas clássicas de otimização inviáveis para a resolução de tais problemas.

A maioria dos problemas do mundo real são voltados para a resolução de problemas com mais de um objetivo, muitos deles conflitantes. Tais problemas são conhecidos como problemas de otimização multiobjetivo (MOOP - Multi Objective Optimization Problem).

Um MOOP é responsável pela resolução de problemas com um conjunto de funções cuja resolutividade, geralmente, é dita conflitante.AE's que resolvem problemas de caráter MOOP, são denominados MOEA (Multi Objective Evolutionary Algorithm). Neste trabalho, foi utilizado o MOEA NSGA-II (Elitist Non-Dominated Sorting Genetic Algorithm), Deb et al. (2000).

O algoritmo NSGA-II utiliza o conceito de crowding distance para calcular o melhor conjunto de soluções conflitantes de um determinado MOOP, evoluindo as soluções para um conjunto de soluções otimizadas ao final do processo. A este conjunto denomina-se conjunto Pareto Otimizado.

\section{METODOLOGIA PROPOSTA}

A infactibilidade de soluções em AE's na resolução do problema de RSDR é algo inerente a estas heurísticas devido à ação dos operadores genéticos. Outras heurísticas buscam na penalização uma forma de resolução deste problema, Shojaeian and Ghandehari (2013) e Ma et al. (2005).

Neste trabalho, as soluções de um determinado espaço solução foram modeladas por meio de um vetor de binários. Cada posição do vetor representa o estado de uma linha do sistema. Cada linha existente comportou-se com uma chave seccionadora, ou seja, o seu fechamento é responsável pela passagem de corrente naquela linha de distribuição.

A infactibilidade de soluções geradas por AE's no problema de RSDR pode ser entendida quando se olha a codificação binária clássica utilizada para se representar os indivíduos da população dos AE's. Desta forma, uma representação possível para o sistema apresentado na Figura 1 pode ser vista na representação binária na Equação 5 .

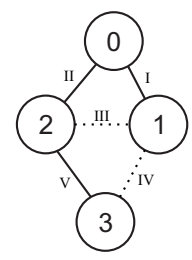

Figura 1. Grafo representando um sistema hipotético.

Os números de 0 a 3 vistos na Figura 1 representam as barras de um determinado sistema de distribuição e as arestas identificadas por I, II, III, IV e V representam as linhas de distribuição interligando as barras. Uma aresta pontilhada identifica uma linha desenergizada. Para o sistema da Figura 1 a solução é representada em 5 .

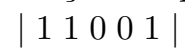

Uma linha com passagem de corrente é representada pelo número 1, enquanto uma chave aberta (linha sem passagem de corrente) é representada pelo número 0 . A ordem das linhas é identificada pelos números I, II, III, IV e $\mathrm{V}$ e esta mesma ordem é utilizada no vetor na Equação 5. A esta representação de 0's e 1's, dá-se o nome de representação binária, Deb et al. (2000).

Uma solução conforme apresentada na Equação 6 é uma solução infactível, haja vista o fato de termos diversos pontos de ilhamentos.

$$
\left|\begin{array}{lllll}
1 & 0 & 0 & 0 & 0
\end{array}\right|
$$

\subsection{Penalização}

A abordagem proposta foi comparada com a metodologia de penalização, Joines and Houck (1994). Seja $S$ uma solução qualquer de uma população $P$ e seja sua função de avaliação adquirida na forma $S$.fitness e a verificação de factibilidade da solução verificada na forma S.factivel, a função de penalização pode ser generalizada no Algoritmo 1 .

\begin{tabular}{|ll|}
\hline Algoritmo 1: Cálculo de fitness S. & $\mathbf{1}$ \\
\hline Entrada: $S$ & \\
if S.factivel then & $\mathbf{2}$ \\
$\mid$ Fitness $=$ S.fitness; & $\mathbf{3}$ \\
else & $\mathbf{4}$ \\
end Fitness $=\operatorname{penaliza}(S) ;$ & $\mathbf{5}$ \\
\hline
\end{tabular}

O Algoritmo 1 é responsável pelo cálculo da função de aptidão (fitness) de uma solução $S$. Duas funções de aptidão são utilizadas neste trabalho: minimização de perda de potência ativa e redução do número de manobras para a RSDR.

$$
\text { Fitness }=\text { S.fitness }+1000
$$

Neste trabalho, a penalização da solução deu-se de duas formas: descarte do indivíduo infactível com posterior substituição deste por uma configuração factível, finalizouse somando-se um valor alto à função de aptidão encontrada (do novo indivíduo) conforme Equação 7.

A representação de uma solução factível em problemas de RSDR pode ser modelada através de grafos, Viana (2017). Devido à versatilidade da topologia malhada dos sistemas de distribuição e de sua operação em árvore, é possível trabalhar com incontáveis topologias de operação.

Embora exista um número muito grande de árvores alcançáveis diante de inúmeras linhas de distribuição, tal fato não é garantidor de que estas obedeçam às restrições de operação exigidas pelo PRODIST em seu módulo 8.

Em SDR's a quantidade de soluções, é proporcional ao número de linhas de distribuição, na forma:

$$
n s=2^{n l}
$$

Com ns representando o número de soluções e $n l$ o total de linhas existentes Para um sistema pequeno por exemplo, de apenas 33 barras e 37 linhas de distribuição, a possibilidade de soluções é de $2^{37}$ conforme Equação 8.

A solução substituta de uma configuração infactível foi gerada a partir do algoritmo de Kruskal, Kruskal (1956). 
O algoritmo de Kruskal gera árvores com base nos pesos de suas arestas utilizando-se do conceito de uma MST (Minimum Spanning Tree, Árvore Espalhada Mínima), Cormen et al. (2009). Os pesos das arestas foram gerados de forma aleatória sempre que a condição da linha 2 do Algoritmo 1 foi falsa.

A estratégia de penalização utilizada descartou soluções infactíveis e em seu lugar inseriu uma nova gerada através do algoritmo de Kruskal.

\subsection{Abordagem de factibilização}

Uma solução infactível dentro do contexto de RSDR representa uma configuração de linhas de distribuição com: barra(s) ilhada(s) e topologia de operação malhada ou fracamente malhada. Um sistema que apresenta as duas características apresentadas acima pode ser visto na Figura 3.

Na Figura 2 a área delimitada cinza representa um espaço factível de buscas para a resolução de um problema fictício. Os pontos demonstram possíveis soluções para o problema. Para este problema as soluções inseridas dentro da área destacada são as soluções factíveis. Qualquer solução fora da figura destacada não representa uma solução viável.

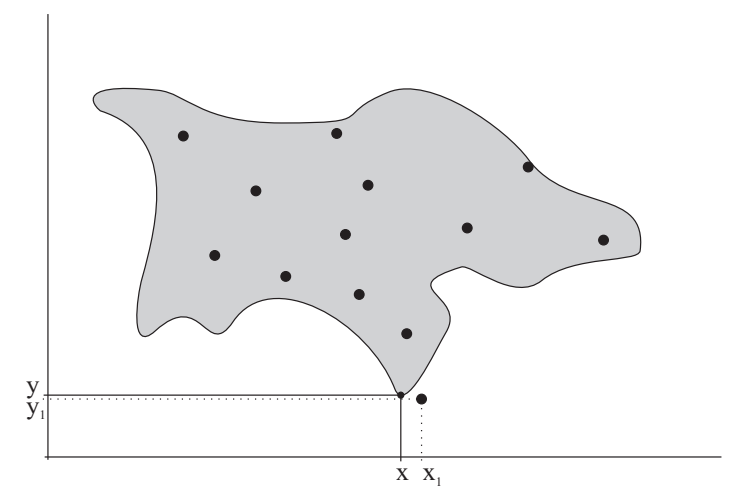

Figura 2. Espaço de busca fictício.

O ponto $(x, y)$ representa o ótimo global deste problema. Se considerarmos a estratégia utilizada até agora, quando o NSGA-II ou qualquer outro MOEA se deparasse com a solução $\left(x_{1}, y_{1}\right)$, a mesma seria descartada imediatamente e substituída por qualquer uma outra solução possível (pontos destacados na área sombreada).

O grafo na Figura 3 representa um sistema de 9 barras, com ciclos e ilhas. As linhas pontilhadas 4-8 e 7-8 representam uma chave aberta.

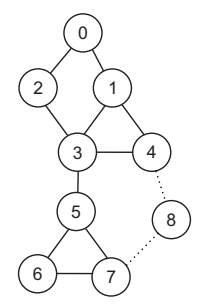

Figura 3. Grafo representando um sistema de 8 barras em malha e com uma barra ilhada.

Uma solução $S$ representada pelo grafo na Figura 3 é dita uma solução infactível para um SDR. Partindo desta solução, pretende-se chegar em uma solução factível, respeitando-se a pré-existência de linhas, não criando novas.

Uma possível factibilização da solução 3 encontra-se na Figura 4. O grafo representado na Figura 4 foi obtido através da heurística de factibilização proposta neste trabalho e é descrita na forma a seguir.

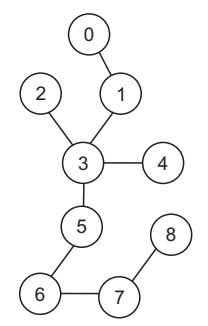

Figura 4. Grafo representando um sistema de 8 barras após factibilização.

Dado um vértice $u$, u.discovered $=$ true significa que o vértice $u$ foi encontrado pela busca; $A d j[u]$ retorna a lista de adjacências de $u ; u . \lambda$ retorna um ponteiro para o pai de $u$. Os Algoritmos 2 e 3 são propostos para o tratamento de infactibilidade.

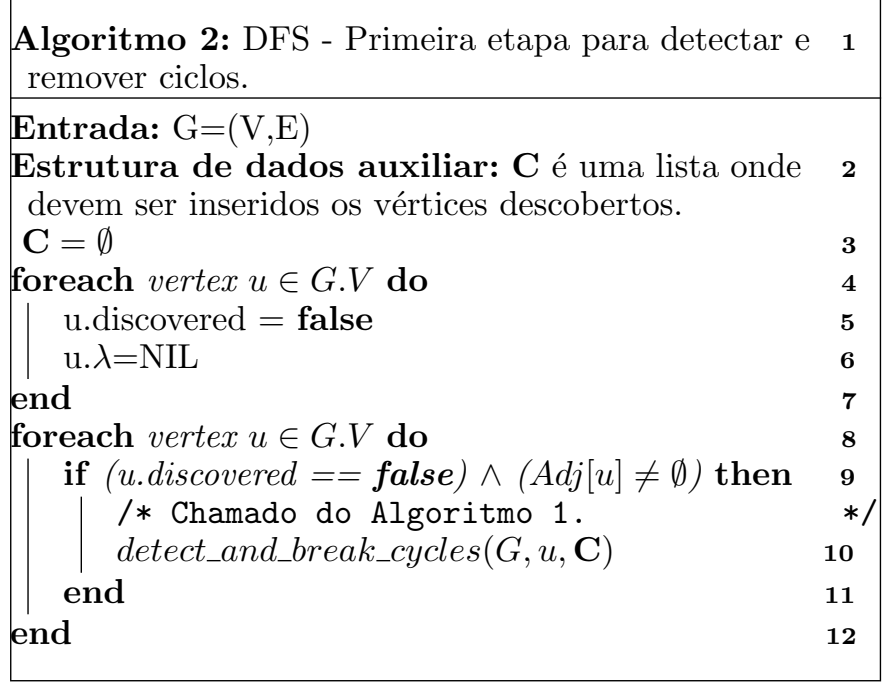

Na linha 8 do Algoritmo 2 visita-se todos os nós(barras) não-ilhadas. Então, se um vértice $v \in G . V$ é um vértice não ilhado, o Algoritmo 3 é chamado e a busca continua.

No Algoritmo 3, deve-se marcar o vértice atual $u$ como visitado e colocá-lo em uma lista C. Se em $A d j[u]$ existir um vértice que está presente em $\mathbf{C}$ e este não é pai de $u$, então um ciclo é detectado.

Quando os Algoritmos 2 e 3 terminarem, todos os vértices não isolados estarão com o status de visitado. Consequentemente, os vértices isolados estarão com o status não visitado. Se existir pelo menos um vértice não visitado, o grafo apresenta ilhamento.

\section{SIMULAÇÕES E RESULTADOS}

O algoritmo NSGA-II proposto neste trabalho foi escrito em $\mathrm{C}++$. Os objetivos (fitness) otimizados foram: o 


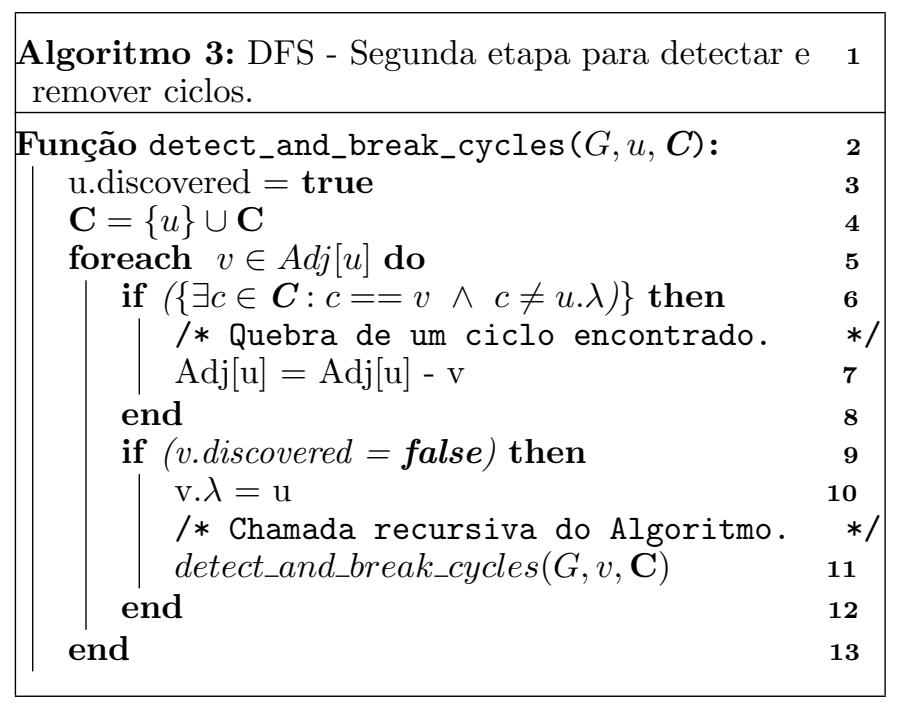

número de manobras e a perda de potência ativa em $k W$. Foram simulados sistemas de 33, 84 e 136 barras. Os dados das barras, linhas e referências dos sistemas podem ser encontrados em Oliveira (2011).

Os resultados para ambos sistemas foram obtidos utilizando os seguintes parâmetros: população de 30 indivíduo, 70 mil avaliações como critério de parada, taxa de mutação obedeceu a Equação 9, taxa de cruzamento 90\%, crossover half uniform, seleção por torneio binário via Deb et al. (2000) baseado no operador crowding distance.

$$
T_{m}=1 / \text { nbits }
$$

Com $T_{m}$ representa a taxa de mutação e nbits é o número de bits necessários para representar um determinado indivíduo.

O indicador de Hypervolume (HV), Deb et al. (2000), é capaz de demonstrar, em um única métrica, os dois principais objetivos da otimização multiobjetivo: a descoberta de soluções mais próximas da Pareto-ótima (convergência) e encontrar as mais diversas soluções possíveis ao longo das soluções não-dominadas obtidas (diversidade). Um algoritmo com o maior HV representa um algoritmo com bom desempenho. A métrica de desempenho utilizada foi o $\mathrm{HV}$ normalizado.

Na Tabela 1 são encontrados os resultados para os sistemas de 33, 84 e 136 barras, destacando-se o valor de $\mathrm{HV}$ e a quantidade de avaliações de fitness necessárias para a obtenção do mesmo.

Tabela 1. Resultados para os sistemas de 33, 84 e 136 barras.

\begin{tabular}{c|c|c|c}
\hline Sistema & Heurística & HV & Avaliações \\
\hline \multirow{2}{*}{33 barras } & Sem & 0.6648 & 39210 \\
\cline { 2 - 4 } & Com & 0.6648 & 2550 \\
\hline \multirow{2}{*}{84 barras } & Sem & 0.6564 & 66060 \\
\cline { 2 - 4 } & Com & 0.7604 & 19170 \\
\hline \multirow{2}{*}{136 barras } & Sem & 0.7910 & 65520 \\
\cline { 2 - 4 } & Com & 0.8416 & 39450 \\
\hline
\end{tabular}

\subsection{Análise da utilização da penalização}

O sistema de 33 barras, teve um HV (0.6649) em um tempo de 73.504 segundos com 39210 avaliações de fitness para sua convergência. Para o sistema de 84 barras foram necessárias 66060 avaliações para a convergência do melhor valor de HV de $0.6564 \mathrm{em}$ um tempo de 185.082 segundos. O sistema de 136 barras apresentou um tempo de 628.089 segundos para sua convergência em 65520 avaliações e um HV de 0.7910 .

\subsection{Análise da aplicação da heurística proposta}

No sistema de 33 barras, para se encontrar o melhor valor de HV (0.6649) foram necessárias 2550 avaliações e um tempo computacional de 33.227 segundos. Para o sistema de 84 barras foram necessárias 19170 avaliações para a convergência do melhor valor de $\mathrm{HV}$ de 0.7604 em um tempo de 80.321 segundos. O último e maior sistema, 136 barras, apresentou um tempo de 134.456 segundos até a convergência de um HV de 0.8416 e um total de 39450.

Um análise comparativa do tempo computacional para os sistemas avaliados pode ser verificada na Figura 5. As barreiras de Pareto Otimizado, Pareto com heurística de factibilização e Pareto com aplicação de penalização podem ser vistas nas Figuras 6,7 e 8 .

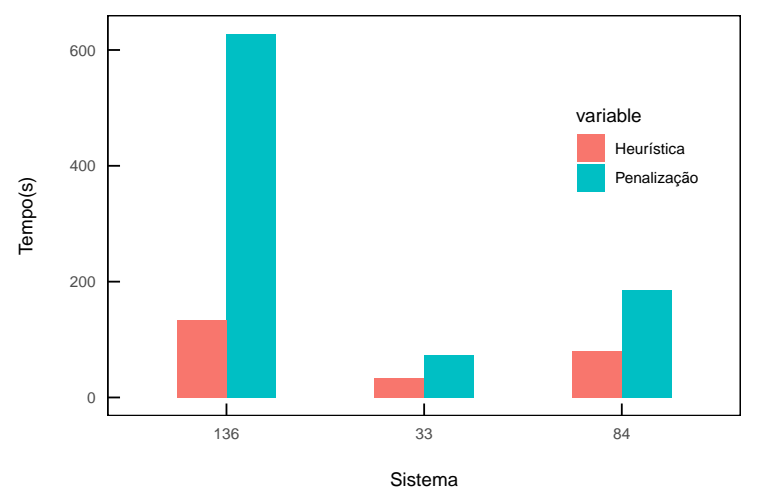

Figura 5. Resultados de tempo computacional para os sistemas 33, 84 e 136 barras.

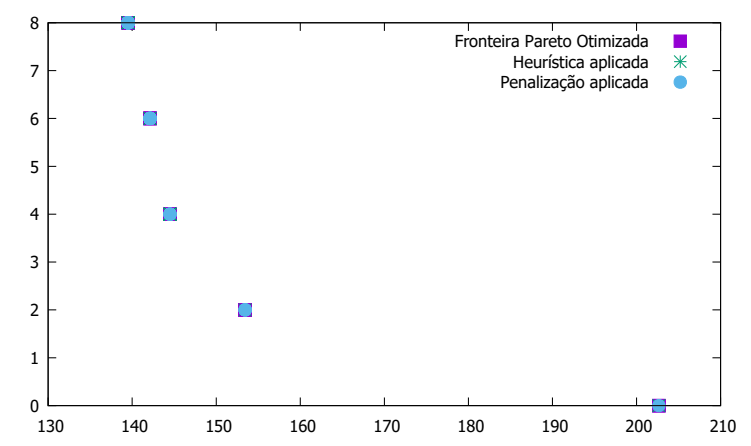

Figura 6. Fronteiras Pareto para o sistema de 33 barras.

O sistema de 33 barras foi o único que apresentou Fronteira de Pareto igual à barreira otimizada, o fato pode ser explicado devido ao sistema ser de pequeno porte, com um número de soluções significativamente inferior quando comparado aos outros sistemas. A aplicação de penalização, apesar de encontrar a mesma barreira de Pareto, encontrou-a com uma significativa diferença de tempo, conforme visto na Figura 5. 


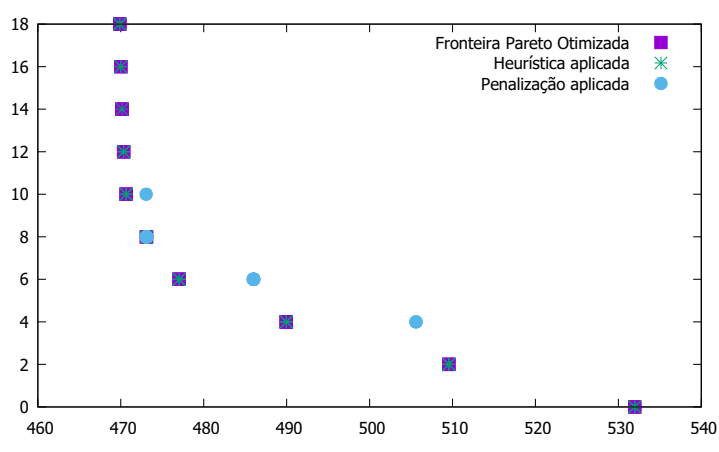

Figura 7. Fronteiras Pareto para o sistema de 84 barras.

Para o sistema de 84 barras, apenas quatro soluções foram encontrados utilizando o método de penalização, e destas, apenas uma apresentou-se na barreira de Pareto otimizado.

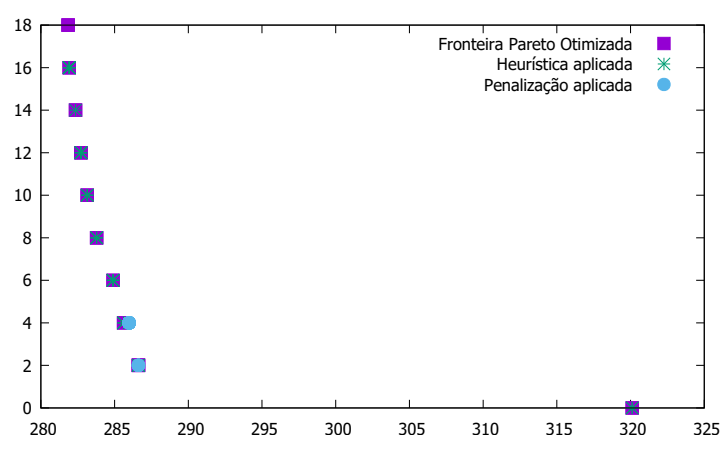

Figura 8. Fronteiras Pareto para o sistema de 136 barras.

Para o sistema de 136 barras apenas 2 soluções foram encontradas para a barreira Pareto utilizando-se o método de penalização, sendo apenas 1 encontrada na barreira otimizada. A barreira encontrada pela heurística de factibilização foi idêntica à encontrada na barreira otimazada.

Em relação ao tempo computacional a aplicação da heurística de factibilização apresentou uma redução de até $78.59 \%$ do tempo computacional necessário para a convergência do indicador de HV. A menor redução encontrada, $54.8 \%$, foi verificada para o sistema de 33 barras.

Além da redução drástica no esforço computacional, as soluções apresentadas em todos os cenários foram idênticas às soluções encontradas na barreira Pareto otimizada.

\section{CONCLUSÕES}

Neste trabalho foi apresentada uma heurística de factibilização para soluções infactíveis provenientes do AEMO NSGA-II no problema de RSDR. A heurística proposta é de simples implementação, de desempenho computacional robusto e de grande eficácia, além de sempre proporcionar soluções factíveis para o problema tratado.

Os resultados encontrados para os três sistemas simulados demonstram grandes diferenças de tempo computacional, chegando o melhor caso a alcançar uma diferença de $78.59 \%$ quando comparado à penalização. As soluções alcançadas, além de apresentar uma grande diferença de tempo, apresentaram um indicador mais elevado nos sistemas de 84 e 136 barras, chegando a um mesmo valor no sistema de 33 barras.

Além da abordagem proposta alcançar tempos computacionais significativamente melhores, a qualidade das soluções propostas, mensuradas pela métrica de HV, foram as melhores em todos os sistemas. Apesar do sistema de menor porte ter aprensentado um mesmo valor na métrica de HV, o tempo computacional mostrou-se muito menor para a heurística de factibilização.

Conclui-se que a heurística de factibilização proposta na resolução do problema de RSDR apresentou um desempenho significativo, com baixo custo computacional envolvido, aprensentado robustez e soluções de excelente qualidade.

\section{REFERÊNCIAS}

Carvalho, M.R. (2006). Estudo comparativo de fluxo de potência para sistemas de distribuição radial. São Carlos, SP. Dissertação (Mestrado em Engenharia Elétrica)-Universidade de São Carlos.

Cormen, T.H., Leiserson, C.E., Rivest, R.L., and Stein, C. (2009). Introduction to algorithms. MIT press.

Deb, K., Agrawal, S., Pratap, A., and Meyarivan, T. (2000). A fast elitist non-dominated sorting genetic algorithm for multi-objective optimization: Nsga-ii. In International conference on parallel problem solving from nature, 849-858. Springer.

Joines, J.A. and Houck, C.R. (1994). On the use of nonstationary penalty functions to solve nonlinear constrained optimization problems with ga's. In Proceedings of the First IEEE Conference on Evolutionary Computation. IEEE World Congress on Computational Intelligence, 579-584. IEEE.

Kruskal, J.B. (1956). On the shortest spanning subtree of a graph and the traveling salesman problem. Proceedings of the American Mathematical society, 7(1), 48-50.

Ma, F., Knight, J.P., and Plett, C. (2005). Physical resource binding for a coarse-grain reconfigurable array using evolutionary algorithms. IEEE Transactions on Very Large Scale Integration (VLSI) Systems, 13(5), 553-563.

Oliveira, M.B.C.d. (2011). Reconfiguração de alimentadores em sistemas de distribuição usando a metaheurística grasp.

Shirmohammadi, D., Hong, H.W., Semlyen, A., and Luo, G. (1988). A compensation-based power flow method for weakly meshed distribution and transmission networks. IEEE Transactions on power systems, 3(2), 753-762.

Shojaeian, S. and Ghandehari, E. (2013). A heuristic multiobjective method for radial distribution networks reconfiguration. Chinese Journal of Engineering.

Su, C.T., Chang, C.F., and Chiou, J.P. (2005). Distribution network reconfiguration for loss reduction by ant colony search algorithm. Electric Power Systems Research, 75(2-3), 190-199.

Viana, Enio R., S.A.S. (2017). Heurística para geração de configurações radiais não ilhadas em sistemas de distribuição. X Encontro Unificado de Computação, $336-344$. 